\title{
Prokhorov distance with rates of convergence under sublinear expectations ${ }^{1)}$
}

\author{
QIANQIAN ZHOU*, ALEXANDER SAKHANENKO** AND JUNYI GUO***
}

\begin{abstract}
Prokhorov distances under sublinear expectations are presented in CLT and functional CLT, and the convergence rates for them are obtained by Lindeberg method. In particular, the obtained estimate in functional CLT yields known Borovkov's estimate in classical functional CLT with explicit constant.
\end{abstract}

Keywords Sublinear expectation; Prokhorov distance; Lindeberg method Mathematics Subject Classification (2010) Primary: 60F05

\section{Introduction}

1.1. Convergence of a sequence of random variables is an important concept in probability theory and its applications. Convergence in distribution is the weakest form of such convergences. However, convergence in distribution is very frequently used in practice, one of the most important application is the central limit theorem (CLT). It is well known that if we have classical i.i.d. random variables $X_{1}, X_{2}, \ldots$ with

$$
\mathbf{E}\left[X_{1}\right]=\mu, \quad 0<\sigma^{2}=\operatorname{Var} X_{1}=\mathbf{E}\left[\left(X_{1}-\mu\right)^{2}\right]<\infty,
$$

then (all limits in the paper are taken as $n \longrightarrow \infty$ )

$$
W_{n}:=\frac{1}{\sqrt{n}} \sum_{i=1}^{n} \frac{X_{i}-\mu}{\sigma} \Rightarrow Z \sim N(0,1),
$$

where $Z \sim N(0,1)$ means that $Z$ has the standard normal distribution. In particular, convergence (2) is equivalent to the fact that for all bounded and

\footnotetext{
* School of Mathematical Sciences, Nankai University, Tianjin 300071, P.R. China. Email: qianqzhou@yeah.net.

** Sobolev Institute of Mathematics, 4 Acad. Koptyug avenue, Novosibirsk 630090, Russia.

School of Mathematical Sciences, Nankai University, Tianjin 300071, P.R. China. Email: aisakh@mail.ru.

*** School of Mathematical Sciences, Nankai University, Tianjin 300071, P.R. China. Email: jyguo@nankai.edu.cn.

1) This work was supported by the National Natural Science Foundation of China (Grant No. 11571189) and the Fundamental Research Fund for the Central Universities.

The research of A.Sakhanenko was also supported by the program of fundamental scientific researches of the SB RAS I.1.3., project 0314-2019-0008
} 
continuous function $\varphi(\cdot)$ we have

$$
\mathbf{E}\left[\varphi\left(W_{n}\right)\right] \longrightarrow \mathbf{E}[\varphi(Z)] .
$$

And (2) is also equivalent to the convergence

$$
\pi\left(W_{n}, Z\right) \rightarrow 0,
$$

where $\pi\left(W_{n}, Z\right)$ is the Prokhorov distance between the distributions in $\mathbb{R}$ of random variables $W_{n}$ and $Z$. We may define this Prokhorov distance in the following way:

$$
\pi\left(W_{n}, Z\right)=\inf \left\{\varepsilon>0: \sup \left[\mathbf{P}\left(W_{n} \in A\right)-\mathbf{P}\left(Z \in A^{\varepsilon}\right)\right] \leq \varepsilon\right\},
$$

where the supremum is taken over all measurable sets $A \subset \mathbb{R}$, and $A^{\varepsilon}$ denotes the $\varepsilon$-neighborhood of $A$, i.e., $A^{\varepsilon}=\{x \in \mathbb{R}: \exists y \in A$ with $|x-y|<\varepsilon\}$.

1.2. The convergence in distribution of a sequence of random processes in the functional central limit theorem has similar properties. Consider again classical i.i.d. random variables from (11) and introduce into consideration the classical random broken line $S(\cdot)$ with the values

$$
S(0)=0, \quad S(k)=\sum_{i=1}^{k} \xi_{i}, \quad \xi_{i}:=\frac{X_{i}-\mu}{\sigma}, \quad i, k=1,2, \ldots
$$

Thus, $S(t)=S([t])+(t-[t]) \xi_{[t]+1}$ is a linear function on each interval of the form $[k, k+1], \quad k=0,1,2, \ldots$. Also introduce the sequence of rescaled broken lines $S_{n}(t)=S(n t) / \sqrt{n}, t \in[0,1], n=1,2, \ldots$ Hence, $S_{n}(\cdot)$ is a broken line with the following values at key points:

$$
S_{n}(k / n)=\frac{1}{\sqrt{n}} \sum_{i=1}^{k} \xi_{i}, \quad \xi_{i}=\frac{X_{i}-\mu}{\sigma}, \quad i, k=1,2, \ldots, n .
$$

We treat $S_{n}=S_{n}(\cdot)$ as a random process with values in the space $\mathbb{C}[0,1]$ of all continuous functions, defined on the interval $[0,1]$, with the uniform norm $\|\cdot\|$. Then the sequence $S_{n}=S_{n}(\cdot)$ satisfies functional CLT, introduced in [2] and [8, i.e., for any real bounded and continuous functional $f(\cdot)$, defined on $\mathbb{C}[0,1]$, we have

$$
\mathbf{E}\left[f\left(S_{n}\right)\right] \longrightarrow \mathbf{E}[f(B)],
$$

where $B=B(\cdot)$ is a Wiener process. This fact is also equivalent to the following convergence

$$
\Pi\left(S_{n}, B\right) \longrightarrow 0,
$$

where $\Pi\left(S_{n}, B\right)$ is the Prokhorov distance between the distributions in $\mathbb{C}[0,1]$ of the processes $S_{n}$ and $B$, i.e.,

$$
\Pi\left(S_{n}, B\right)=\inf \left\{\varepsilon>0: \sup \left[\mathbf{P}\left(S_{n} \in D\right)-\mathbf{P}\left(B \in D^{\varepsilon}\right)\right] \leq \varepsilon\right\},
$$

where the supremum is taken over all measurable sets $D \subset \mathbb{C}[0,1]$, and $D^{\varepsilon}$ denotes the $\varepsilon$-neighborhood of $D$, i.e.,

$$
D^{\varepsilon}=\{x \in \mathbb{C}[0,1]: \exists y \in D \quad \text { with } \quad\|x-y\|<\varepsilon\},
$$


where $\|x-y\|=\max \{|x(t)-y(t)|: t \in[0,1]\}$ is the distance between $x, y \in$ $\mathbb{C}[0,1]$.

1.3. Prokhorov distance was introduced in the seminal paper 8 of Prokhorov as an useful instrument for investigation of convergence of probability distributions in separable metric spaces. It is also an useful instrument for investigations of rates of convergence in functional CLT. Indeed, if the functional $f(\cdot)$ is Lipschitz continuous, i.e.,

$$
\forall x, y \in \mathbb{C}[0,1] \quad|f(x)-f(y)| \leq L\|x-y\|, \quad L<\infty
$$

and if $f(B)$ has a bounded density, i.e.,

$$
\forall a \in \mathbb{R} \quad \forall h \geq 0 \quad \mathbf{P}(a \leq f(B) \leq a+h) \leq K h, \quad K<\infty,
$$

then (see [1] )

$$
\sup _{a}\left|\mathbf{P}\left(f\left(S_{n}\right) \leq a\right)-\mathbf{P}(f(B) \leq a)\right| \leq(K L+1) \Pi\left(S_{n}, B\right) .
$$

Inspired by inequality (8), a difficult task of obtaining estimates for the Prokhorov distance $\Pi\left(S_{n}, B\right)$ attracted a lot of attention. The first such estimate, when $\mathbf{E}\left|\xi_{1}\right|^{3}<\infty$, was obtained by Prokhorov in [8]. The first general estimate due to Borovkov [1]. For i.i.d. random variables $\left\{\xi_{i}\right\}$ he proved in [1] that

$$
\Pi\left(S_{n}, B\right) \leq C_{1}(p) \frac{\left(\mathbf{E}\left|\xi_{1}\right|^{p}\right)^{1 /(p+1)}}{n^{(p-2) /(2 p+2)}}, \quad p \in[2,3],
$$

where $C_{1}(p)$ depends only on $p$ and $\sup _{p \in[2,3]} C_{1}(p)<\infty$ is an absolute constant. Later Sakhanenko in [10] extended (9]) for all $p \geq 2$. In [10] and 12] you may find simple proofs of the fact that inequality (9) is unimprovable for all $p \geq 2$, up to a constant $C_{1}(p)$.

1.4. In the above statements the probability $\mathbf{P}$ and expectation $\mathbf{E}$ are considered in classical sense. Recently, motivated by the risk measures, superhedge pricing and modeling uncertain in finance, Peng in [4] (see, also [5] and 7]) introduces the notion of sublinear expectation. Thus, instead of $\mathbf{P}$ and $\mathbf{E}$ in the classical sense, we consider in this paper the sublinear expectation $\overline{\mathbf{E}}$, which is a functional defined on a linear space $\mathcal{H}$ and for each $X, Y \in \mathcal{H}$ the following properties are satisfied:

(i) monotonicity: $\overline{\mathbf{E}}[X] \leq \overline{\mathbf{E}}[Y]$ if $X \leq Y$;

(ii) constant preserving: $\overline{\mathbf{E}}[c]=c$ for all constants $c \in \mathbb{R}$;

(iii) sub-additivity: $\overline{\mathbf{E}}[X+Y] \leq \overline{\mathbf{E}}[X]+\overline{\mathbf{E}}[Y]$;

(iv) positive homogeneity: $\overline{\mathbf{E}}[\lambda X]=\lambda \overline{\mathbf{E}}[X]$ for all constants $\lambda \geq 0$. The triple $(\Omega, \mathcal{H}, \overline{\mathbf{E}})$ is called a sublinear expectation space.

As a standard example, consider a sequence $\xi_{1}, \xi_{2}, \ldots$ of classical i.i.d. random variables defined on a probability space $\left(\Omega, \mathcal{F}, \mathbf{P}_{\theta}\right)$ with $\theta \in \Theta$, where $\Theta$ contains more than one element. Denote by $\Psi_{n}$ the set of all bounded and continuous functions $\psi(\cdot): \mathbb{R}^{n} \rightarrow \mathbb{R}$, and with $\vec{\xi}_{n}=\left(\xi_{1}, \ldots, \xi_{n}\right)$ introduce:

$$
\mathcal{H}:=\left\{\psi\left(\vec{\xi}_{n}\right): \psi(\cdot) \in \Psi_{n}\right\} \quad \text { and } \quad \overline{\mathbf{E}} \psi\left(\vec{\xi}_{n}\right):=\sup _{\theta \in \Theta} \mathbf{E}_{\theta} \psi\left(\vec{\xi}_{n}\right) .
$$

Then the triple $(\Omega, \mathcal{H}, \overline{\mathbf{E}})$ defined in (10) is a sublinear expectation space. 
For other examples see 4 and 7 .

If we want to obtain CLT or results about Prokhorov distance under sublinear expectation then we need a definition of independence. Similar to Peng [5] and [7, we say that a random vector $\vec{Y} \in \mathbb{R}^{l}$ is independent to another random vector $\vec{X} \in \mathbb{R}^{m}$ if

$$
\psi(\vec{X}, \vec{Y}) \in \mathcal{H} \quad \text { and } \quad \overline{\mathbf{E}}[\psi(\vec{X}, \vec{Y})]=\overline{\mathbf{E}}\left[\left.\overline{\mathbf{E}}[\psi(\vec{x}, \vec{Y})]\right|_{\vec{x}=\vec{X}}\right]
$$

for all bounded and continuous function $\psi(\cdot): \mathbb{R}^{l+m} \rightarrow \mathbb{R}$. This independence means that the distribution of $\vec{Y}$ does not change after any realization $\vec{x}$ of $\vec{X}$.

A sequence of random variables $X_{1}, X_{2}, \ldots$ is said to be i.i.d. with $X_{0}$, if for each $k=2,3, \ldots$ random variable $X_{k}$ is independent to the vector $\vec{X}_{k-1}=$ $\left(X_{1}, X_{2}, \ldots, X_{k-1}\right)$ and if $X_{k}$ is identically distributed with $X_{0}$ for all $k \geq 1$. The latter means that

$$
\forall k \geq 1 \overline{\mathbf{E}}\left[\psi\left(X_{k}\right)\right]=\overline{\mathbf{E}}\left[\psi\left(X_{0}\right)\right] \text { for any continuous and bounded } \psi(\cdot) .
$$

Thus, for a sequence of i.i.d. with $X_{0} \in \mathcal{H}$ random variables, under a sublinear expectation $\overline{\mathbf{E}}$, we have two boundaries for values of expectations:

$$
-\infty<\underline{\mu}=\underline{\mathbf{E}}\left[X_{0}\right]=-\overline{\mathbf{E}}\left[-X_{0}\right] \leq \bar{\mu}=\overline{\mathbf{E}}\left[X_{0}\right]<\infty .
$$

Here and later on we use notation $\underline{\mathbf{E}}[X]:=-\overline{\mathbf{E}}[-X]$ for all $X \in \mathcal{H}$. And when $X_{0}^{2} \in \mathcal{H}$, instead of variance we have two functions:

$$
0 \leq \underline{\sigma}^{2}(\mu)=\underline{\mathbf{E}}\left[\left(X_{0}-\mu\right)^{2}\right] \leq \bar{\sigma}^{2}(\mu)=\overline{\mathbf{E}}\left[\left(X_{0}-\mu\right)^{2}\right]<\infty, \quad \mu \in[\underline{\mu}, \bar{\mu}] .
$$

Later on in the paper we everywhere assume that

$$
X_{0}, X_{0}^{2} \in \mathcal{H} \quad \text { and } \quad 0<\underline{\sigma}:=\inf _{\mu \in[\underline{\mu}, \bar{\mu}]} \underline{\sigma}(\mu) \leq \bar{\sigma}:=\sup _{\mu \in[\underline{\mu}, \bar{\mu}]} \bar{\sigma}(\mu)<\infty .
$$

1.5. The situation with CLT under sublinear expectation is essentially more difficult, even in the case when

$$
\underline{\mu}=\bar{\mu}=0, \quad 0<\underline{\sigma}(0) \leq \bar{\sigma}(0)<\infty, \quad\left|X_{0}\right|^{3} \in \mathcal{H} .
$$

Introduce a class of real functions satisfying the Hölder condition:

$$
L_{1}(\varphi):=\sup \{|\varphi(x+\delta)-\varphi(x)| / \delta: \delta>0, \quad x \in \mathbb{R}\}<\infty .
$$

In [6] and 7] Peng presented the CLT for a sequence of i.i.d. random variables under sublinear expectations. Under assumptions (16) and (17) he proved that

$$
\overline{\mathbf{E}} \varphi\left(\bar{W}_{n}\right) \longrightarrow G(\varphi, c) \quad \text { for } \quad \bar{W}_{n}=\frac{1}{\bar{\sigma}(0) \sqrt{n}} \sum_{i=1}^{n} X_{i}, \quad c=\frac{\sigma(0)}{\bar{\sigma}(0)},
$$

where the functional $G(\varphi, c)$ may be described in terms of so called viscosity solutions of parabolic partial differential equations. Only when $0<\underline{\sigma}(0)=\bar{\sigma}(0)$ the functional $G(\varphi, 1)=\mathbf{E} \varphi(Z)$ coincides with the right hand side in (3).

The nature of many difficulties in understanding of CLT under sublinear expectation was essentially clarified in the remarkable work 3 by Fang, Peng, 
Shao and Song. They suggested to consider normalized sums of the following form

$$
W_{n, n}:=\frac{1}{\sqrt{n}} \sum_{i=1}^{n} \frac{X_{i}-\mu_{i}}{\sigma_{i}}
$$

where $\mu_{i}$ and $\sigma_{i}$ are special functions of $\vec{X}_{i-1}$ which may depend also on numbers $i, n, \varepsilon$ and on the function $\varphi$ with condition (17). For special class of sublinear expectations with many additional technical assumptions, including (10) and (15), they proved in 3 , that

$$
\overline{\mathbf{E}} \varphi\left(W_{n, n}\right)-\mathbf{E} \varphi(Z)=L_{1}(\varphi) O\left(\frac{\log n}{\sqrt{n}}\right),
$$

where $Z$ is again the standard Gaussian random variable. We emphasize that the main advantage of (20) in comparison with (18) consists in the fact that the limit $\mathbf{E} \varphi(Z)$ in (20) is exactly the same as in the classical CLT.

We are going to continue investigations from [3], but under general definition of sublinear expectations. And we will consider below expectations of noncontinuous indicators instead of smooth functions $\varphi$ with condition (17).

1.6. In the present paper we consider a sequence of random variables $X_{1}, X_{2}, \ldots$ in a sublinear expectation space $(\Omega, \mathcal{H}, \overline{\mathbf{E}})$, which are supposed to be i.i.d. with $X_{0}$, satisfying (12) and (15). Our main aim is to generalize the classical Prokhorov distances into subliner expectation space and obtain convergence rates for Prokhorov distances in CLT and functional CLT by appropriate generalizations of Lindeberg method. For the special broken line $S_{n}=S_{n}(\cdot)$ we are going to obtain estimates for the values of the form

$$
\overline{\mathbf{P}}\left(S_{n} \in D\right)-\mathbf{P}\left(B \in D^{\varepsilon}\right), \quad D \subset \mathbb{C}[0,1], \quad \varepsilon>0,
$$

where $B=B(\cdot)$ is a Wiener process and

$$
\overline{\mathbf{P}}\left(S_{n} \in D\right):=\inf \left\{\overline{\mathbf{E}} \psi\left(\vec{X}_{n}\right): \psi\left(\vec{X}_{n}\right) \geq \mathbf{I}\left(S_{n} \in D\right), \psi\left(\vec{X}_{n}\right) \in \mathcal{H}\right\} \leq 1
$$

since $\mathbf{I}\left(S_{n} \in D\right) \leq 1 \in \mathcal{H}$. Underline that $\psi\left(\vec{X}_{k}\right) \in \mathcal{H}$ for all bounded and continuous functions $\psi(\cdot): \mathbb{R}^{k} \rightarrow \mathbb{R}$; this fact follows from the assumption (11).

The main difference with the classical case is that instead of (4) the random broken line $S_{n}=S_{n}(\cdot)$ has the following special values at key points:

$$
S_{n}\left(\frac{k}{n}\right)=W_{k, n}:=\frac{1}{\sqrt{n}} \sum_{i=1}^{k} \frac{X_{i}-\mu_{i}}{\sigma_{i}}, \quad i, k=0,1,2, \ldots, n,
$$

where $S_{n}(0)=W_{0, n}=0$ and $\mu_{i}=\mu_{i, n, D, \varepsilon}\left(\vec{X}_{i-1}\right)$ and $\sigma_{i}=\sigma_{i, n, D, \varepsilon}\left(\vec{X}_{i-1}\right)$ are special continuous functions of $\vec{X}_{i-1}$ which may depend also on numbers $i, n, \varepsilon$ and on the set $D$ from (21). In addition

$$
\underline{\mu} \leq \mu_{i} \leq \bar{\mu} \quad \text { and } \quad 0<\underline{\sigma} \leq \underline{\sigma}\left(\mu_{i}\right) \leq \sigma_{i} \leq \bar{\sigma}\left(\mu_{i}\right) \leq \bar{\sigma}, \quad i=1,2, \ldots, n .
$$

In Section 2 we introduce Prokhorov distances in sublinear expectation spaces and present estimates for them as for one-dimensional CLT, so for functional CLT. Underline, that our estimates from Theorem 1in functional CLT is, 
in some sense, better than unimprovable Borovkov's estimate (9). Indeed, for the classical Prokhorov distance under standard assumption (1) our Theorem 1 yields, for $p \in[2,3]$, the following inequality

$$
\Pi\left(S_{n}, B\right) \leq 4.7 \frac{\left(\mathbf{E} \min \left\{\left|\xi_{1}\right|^{p}, \sqrt{n} \xi_{1}^{2}\right\}\right)^{1 /(p+1)}}{n^{(p-2) /(2 p+2)}} \leq 4.7 \frac{\left(\mathbf{E}\left|\xi_{1}\right|^{p}\right)^{1 /(p+1)}}{n^{(p-2) /(2 p+2)}} .
$$

Thus, we obtained Borovkov's estimate (9) with constant $C_{1}(p)=4.7$ for $p \in$ $[2,3]$. As far as we know it is the first case when an estimate in the classical functional CLT is obtained with an explicit numerical constant (about previous attempt see Remark 5 below). Moreover, the first inequality in (25) is even better, because it uses truncated moment and does not require the existence of moments, but variance.

1.7. The rest of the paper is organized as follows. The main results about Prokhorov distances defined on sublinear expectation spaces are presented in Section 2. In Sections 3 and 4 we prove our main Theorem 1 and its corollaries. In section 5 we prove simpler Theorem 2 with estimates in one-dimensional CLT.

\section{$2 \quad$ Main results}

2.1. Preliminary agreements and notations. Later on for any function $\phi(\cdot): \mathbb{R} \rightarrow \mathbb{R}$ we use notation

$$
\overline{\mathbf{E}}\left[\phi\left(X_{0}\right)\right]:=\inf \left\{\overline{\mathbf{E}}[X]: X \geq \phi\left(X_{0}\right), X \in \mathcal{H}\right\} \leq \infty,
$$

where we assume that $\inf \emptyset=\infty$. Now for $\mu \in[\mu, \bar{\mu}], p \geq 2$ and $C>0$ define

$$
\xi_{0}(\mu):=\left(X_{0}-\mu\right) / \underline{\sigma}(\mu) \text { and } \gamma_{p}(\mu, C):=\overline{\mathbf{E}}\left[\left|\xi_{0}(\mu)\right|^{p} \wedge C^{p-2} \xi_{0}^{2}(\mu)\right],
$$

where $a \wedge b$ means $\min \{a, b\}$. Introduce

$$
\gamma_{p}(C):=\sup _{\mu \in \underline{\mu}, \bar{\mu}]} \gamma_{p}(\mu, C) \leq C^{p-2} \bar{\sigma}^{2} / \underline{\sigma}^{p}<\infty, \quad C>0, \quad p \geq 2 .
$$

Define also

$$
\sup _{C>0} \gamma_{p}(C) \leq \gamma_{p}:=\sup _{\mu \in[\underline{\mu}, \bar{\mu}]} \overline{\mathbf{E}}\left[\left|\xi_{0}(\mu)\right|^{p}\right] \leq \infty, \quad p \geq 2 .
$$

Remark 1. We treat the distribution of $X_{0}$ as a specially chosen one in the class of distributions, which satisfy (12) and (15), and which approximately minimize in this class the value $\gamma_{p}$ or $\gamma_{p}(C)$ for some $p$ and $C$. In particular, we do not assume, for example, that $\overline{\mathbf{E}} X_{i}^{2}<\infty$ for some $i \neq 0$.

2.2. Estimates in functional CLT. For each $i \geq 1$ introduce into consideration the set $\mathcal{M}(i)$ of all pares $\left(\mu_{i}, \sigma_{i}\right)$ which are continuous functions of $\vec{X}_{i-1}$ and satisfy conditions (24). And denote by $\mathcal{M}_{n}$ the set $\left\{\left(\mu_{i}, \sigma_{i}\right) \in \mathcal{M}(i), i=\right.$ $1, \ldots, n\}$ of all sequences of length $n$ of such pares, which are essentially used in construction (23) of random broken line $S_{n}$.

Theorem 1. Under assumptions (15) for each set $D \subset \mathbb{C}[0,1]$ and any $\varepsilon>0$ there exist values $\left\{\left(\mu_{i}, \sigma_{i}\right)\right\} \in \mathcal{M}_{n}$ which may depend on $D, \varepsilon, n$ and such that

$$
\overline{\mathbf{P}}\left(S_{n} \in D\right)-\mathbf{P}\left(B \in D^{\varepsilon}\right) \leq \tilde{\Pi}_{n}(\varepsilon, p):=\frac{C_{2}(p) \gamma_{p}(\varepsilon \sqrt{n})}{n^{(p-2) / 2} \varepsilon^{p}} \leq \frac{C_{2}(p) \gamma_{p}}{n^{(p-2) / 2} \varepsilon^{p}},
$$

where $p \in[2,3]$ and $C_{2}(p):=\min \left\{184,(4.7)^{p+1}\right\}$. 
In other words, we proved in (28) that for $p \in[2,3]$

$$
\bar{\Delta}_{n}(D, \varepsilon):=\inf _{\left\{\left(\mu_{i}, \sigma_{i}\right)\right\} \in \mathcal{M}_{n}} \overline{\mathbf{P}}\left(S_{n} \in D\right)-\mathbf{P}\left(B \in D^{\varepsilon}\right) \leq \tilde{\Pi}_{n}(\varepsilon, p) .
$$

Introduce notation:

$$
\bar{\Pi}\left(S_{n}, B\right)=\inf \left\{\varepsilon>0: \sup _{D \subset \mathbb{C}[0,1]} \bar{\Delta}_{n}(D, \varepsilon) \leq \varepsilon\right\} .
$$

Later on we consider the value $\bar{\Pi}\left(S_{n}, B\right)$ as Prokhorov distance between distributions in $\mathbb{C}[0,1]$ of $S_{n}$ and $B$ defined on a sublinear expectation space.

Corollary 1. Under conditions of Theorem 1

$$
\bar{\Pi}\left(S_{n}, B\right) \leq \tilde{\Pi}_{n, p}:=\frac{C_{3}\left(\gamma_{p}(\sqrt{n})\right)^{1 /(p+1)}}{n^{(p-2) /(2 p+2)}} \leq \frac{C_{3} \gamma_{p}^{1 /(p+1)}}{n^{(p-2) /(2 p+2)}},
$$

where $p \in[2,3]$ and $C_{3}=4.7$.

As it was mentioned in introduction, in classical case our estimate 300 for our generalization of Prokhorov distance implies known unimprovable estimate (9) of Borovkov. Note also that our Prokhorov distance satisfies the following analog of an classical property (8).

Corollary 2. Under assumptions of Theorem 1, for each functional $f(\cdot)$ which satisfies conditions (6) and (7) we have

$$
\inf _{\left\{\left(\mu_{i}, \sigma_{i}\right)\right\} \in \mathcal{M}_{n}} \overline{\mathbf{P}}\left(f\left(S_{n}\right) \leq a\right)-\mathbf{P}(f(B) \leq a) \leq(K L+1) \tilde{\Pi}_{n, p} .
$$

2.3. Estimates in one-dimensional CLT. For every $i \geq 1$ introduce the set $\mathcal{A}(i)$ of all pares $\left(\mu_{i}, \sigma_{i}\right)$ which are (one-dimensional) continuous functions of $W_{i-1, n}$ and satisfy conditions (24). Denote by $\mathcal{A}_{n}$ the set $\left\{\left(\mu_{i}, \sigma_{i}\right) \in \mathcal{A}(i), i=\right.$ $1, \ldots, n\}$ of all sequences of length $n$ of such pares.

Theorem 2. Under assumptions (15) for each set $A \subset \mathbb{R}$ and any $\varepsilon>0$ there exist values $\left\{\left(\mu_{i}, \sigma_{i}\right)\right\} \in \mathcal{A}_{n}$ which may depend on $A, \varepsilon, n$ and such that

$$
\overline{\mathbf{P}}\left(W_{n, n} \in A\right)-\mathbf{P}\left(Z \in A^{\varepsilon}\right) \leq \frac{C_{4} \gamma_{p}(\varepsilon \sqrt{n})}{n^{(p-2) / 2} \varepsilon^{p}} \leq \frac{C_{4} \gamma_{p}}{n^{(p-2) / 2} \varepsilon^{p}}, \quad p \in[2,3],
$$

where $Z \sim N(0,1)$ and $C_{4}=42<(3.5)^{p+1}$. In addition, when $\gamma_{3}<\infty$ we also have

$$
\overline{\mathbf{P}}\left(W_{n, n} \in A\right)-\mathbf{P}\left(Z \in A^{\varepsilon}\right) \leq \frac{C_{5} \gamma_{3}}{\varepsilon \sqrt{n}} \quad \text { with } \quad C_{5}=12 .
$$

It is equivalent to say that we obtained in (32) and (33) estimates for the value

$$
\bar{\delta}_{n}(A, \varepsilon):=\inf _{\left\{\left(\mu_{i}, \sigma_{i}\right)\right\} \in \mathcal{A}_{n}} \overline{\mathbf{P}}\left(W_{n, n} \in A\right)-\mathbf{P}\left(Z \in A^{\varepsilon}\right) .
$$

Introduce notation:

$$
\bar{\pi}\left(W_{n, n}, Z\right)=\inf \left\{\varepsilon>0: \sup _{A \subset \mathbb{R}} \bar{\delta}_{n}(A, \varepsilon) \leq \varepsilon\right\} .
$$


Later on we treat $\bar{\pi}\left(W_{n, n}, Z\right)$ as Prokhorov distance between distributions in $\mathbb{R}$ of $W_{n, n}$ and $Z$ defined on a sublinear expectation space.

We have immediately from (33) and (34) that

$$
\bar{\pi}\left(W_{n, n}, Z\right) \leq \sqrt{C_{5} \gamma_{3}} / n^{1 / 4} .
$$

Remark 2. For classical Prokhorov distance $\pi\left(W_{n}, Z\right)$ in classical CLT for i.i.d. random variables under assumptions (1) Yurinsky [13] obtained the following estimate

$$
\pi\left(W_{n}, Z\right) \leq C_{6} \mathbf{E}\left|\xi_{1}\right|^{3} / \sqrt{n}, \quad \xi_{1}:=\left(X_{1}-\mu\right) / \sigma,
$$

where $C_{6}$ is an absolute constant. Unfortunately, we do not see a method to obtain such sharp estimate for $\bar{\pi}\left(W_{n, n}, Z\right)$, instead of (35).

Underline also that in Theorems 1 and 2 we never supposed that sets $D$ or $A$ be measurable.

The rest of the paper is devoted to proofs of results presented above.

\section{Main lemmas}

3.1. Special functions. Let $X_{0}$ be a random variable under sublinear expectation which satisfies condition (15). Then for all twice continuously differentiable function $h(\cdot)$ and for all non-random $\varkappa>0$ we may introduce the following functions:

$$
\alpha_{\varkappa}(x \mid h):=\frac{\bar{u}+\underline{\mu}}{2}+\frac{\bar{u}-\underline{\mu}}{2} \cdot \frac{h^{\prime}(x)}{\varkappa}, \quad \text { if } \quad\left|h^{\prime}(x)\right| \leq \varkappa ;
$$

and when $\left|h^{\prime}(x)\right|>\varkappa$ we put

$$
\alpha_{\varkappa}(x \mid h):=\left\{\begin{array}{l}
\bar{\mu}, \text { if } h^{\prime}(x)>\varkappa, \\
\underline{\mu}, \text { if } h^{\prime}(x)<-\varkappa .
\end{array}\right.
$$

It is easy to see that $\alpha_{\varkappa}(x \mid h)$ is continuous for all $x \in \mathbb{R}$, since it is a continuous function of $h^{\prime}(x)$.

For all non-random $\kappa>0$ introduce

$\beta_{\varkappa, \kappa}^{2}(x \mid h):=\frac{\bar{\sigma}^{2}\left(\alpha_{\varkappa}(x \mid h)\right)+\underline{\sigma}^{2}\left(\alpha_{\varkappa}(x \mid h)\right)}{2}+\frac{\bar{\sigma}^{2}\left(\alpha_{\varkappa}(x \mid h)\right)-\underline{\sigma}^{2}\left(\alpha_{\varkappa}(x \mid h)\right)}{2} \cdot \frac{h^{\prime \prime}(x)}{\kappa}$,

when $\left|h^{\prime \prime}(x)\right| \leq \kappa$; and if $\left|h^{\prime \prime}(x)\right|>\kappa$ we put

$$
\beta_{\varkappa, \kappa}^{2}(x \mid h):=\left\{\begin{array}{l}
\bar{\sigma}^{2}\left(\alpha_{\varkappa}(x \mid h)\right), \text { if } h^{\prime \prime}(x)>\kappa, \\
\underline{\sigma}^{2}\left(\alpha_{\varkappa}(x \mid h)\right), \text { if } h^{\prime \prime}(x)<-\kappa .
\end{array}\right.
$$

It is easy to see that $\beta_{\varkappa, \kappa}(x \mid h)$ is also continuous for all $x \in \mathbb{R}$, because it is a continuous function of $h^{\prime \prime}(x)$ and $h^{\prime}(x)$. In addition, for all $x \in \mathbb{R}$

$\underline{\mu} \leq \alpha_{\varkappa}(x \mid h) \leq \bar{\mu} \quad$ and $\quad 0<\underline{\sigma} \leq \underline{\sigma}\left(\alpha_{\varkappa}(x \mid h)\right) \leq \beta_{\varkappa, \kappa}(x \mid h) \leq \bar{\sigma}\left(\alpha_{\varkappa}(x \mid h)\right) \leq \bar{\sigma}$.

For any $\varkappa, \kappa>0$ and $x \in \mathbb{R}$ define

$$
\xi(x)=\left(X_{0}-\alpha_{\varkappa}(x \mid h)\right) / \beta_{\varkappa, \kappa}(x \mid h) ; \quad \text { so that }|\xi(x)| \leq\left|\xi_{0}\left(\alpha_{\varkappa}(x \mid h)\right)\right| .
$$


Lemma 1. If $h(\cdot)$ is a twice continuously differentiable function, then for all $\varkappa, \kappa>0$ and $x \in \mathbb{R}$

$$
\begin{aligned}
\overline{\mathbf{E}} h\left(x+\frac{\xi(x)}{\sqrt{n}}\right) & \leq \mathbf{E} h\left(x+\frac{Z}{\sqrt{n}}\right)+\frac{\varkappa(\bar{\mu}-\underline{\mu})}{2 \sqrt{n} \underline{\sigma}}+\frac{\kappa}{2 n}\left(\frac{\bar{\sigma}^{2}}{\underline{\sigma}^{2}}-1\right) \\
& +\overline{\mathbf{E}} \delta\left(x, \frac{\xi(x)}{\sqrt{n}} \mid h\right)-\mathbf{E} \delta\left(x, \frac{Z}{\sqrt{n}} \mid h\right),
\end{aligned}
$$

where classical random variable $Z$ has standard normal distribution and

$$
\delta(x, y \mid h)=h(x+y)-h(x)-y h^{\prime}(x)-y^{2} h^{\prime \prime}(x) / 2 .
$$

Remark 3. If $X_{0}$ is a classical random variable we have

$$
\xi(x)=\frac{X_{0}-\mathbf{E} X_{0}}{\sqrt{\operatorname{Var} X_{0}}}, \quad \mathbf{E} \xi(x)=\mathbf{E} Z=0, \quad \mathbf{E} \xi^{2}(x)=\mathbf{E} Z^{2}=1 .
$$

Hence, instead of (42) we have

$$
\mathbf{E} h\left(x+\frac{\xi(x)}{\sqrt{n}}\right)=\mathbf{E} h\left(x+\frac{Z}{\sqrt{n}}\right)+\mathbf{E} \delta\left(x, \frac{\xi(x)}{\sqrt{n}} \mid h\right)-\mathbf{E} \delta\left(x, \frac{Z}{\sqrt{n}} \mid h\right) .
$$

Equality (44) plays an important role in the Lindeberg method to prove CLT. By this reason we can consider our proof, based on Lemma 1, as a natural generalization of the Lindeberg method for the case of sublinear expectations.

Remark 4. We may define functions $\alpha_{0}(x \mid h)$ and $\beta_{0,0}(x \mid h)$ by formulas (37) and (39) with $\varkappa=\kappa=0$. In this case we may expect that for all $x \in \mathbb{R}$,

$$
\overline{\mathbf{E}}\left[h^{\prime}(x) \xi(x)\right]=0 \quad \text { and } \quad \overline{\mathbf{E}}\left[h^{\prime \prime}(x) \xi^{2}(x)\right]=h^{\prime \prime}(x) .
$$

But functions $\alpha_{0}(x \mid h)$ and $\beta_{0,0}(x \mid h)$ are not continuous. By this reason, in this paper we use more complicated continuous functions $\alpha_{\varkappa}(x \mid h)$ and $\beta_{\varkappa, \kappa}(x \mid h)$ instead of the simpler functions $\alpha_{0}(x \mid h)$ and $\beta_{0,0}(x \mid h)$.

3.2. Proof of Lemma 1. For a fixed $x$ take Taylor expansions of $h\left(x+\frac{Z}{\sqrt{n}}\right)$ at $x$. Then

$$
\begin{gathered}
h\left(x+\frac{Z}{\sqrt{n}}\right)=h(x)+\frac{Z}{\sqrt{n}} h^{\prime}(x)+\frac{Z^{2}}{2 n} h^{\prime \prime}(x)+\delta\left(x, \frac{Z}{\sqrt{n}} \mid h\right), \\
\mathbf{E} h\left(x+\frac{Z}{\sqrt{n}}\right)=h(x)+\frac{1}{2 n} h^{\prime \prime}(x)+\mathbf{E} \delta\left(x, \frac{Z}{\sqrt{n}} \mid h\right) .
\end{gathered}
$$

Similarly,

$$
h\left(x+\frac{\xi(x)}{\sqrt{n}}\right)=h(x)+\frac{\xi(x)}{\sqrt{n}} h^{\prime}(x)+\frac{\xi^{2}(x)}{2 n} h^{\prime \prime}(x)+\delta\left(x, \frac{\xi(x)}{\sqrt{n}} \mid h\right),
$$

and by the properties (ii) - (iv) of sublinear expectation we have

$$
\overline{\mathbf{E}}\left[h\left(x+\frac{\xi(x)}{\sqrt{n}}\right)\right] \leq h(x)+\frac{\overline{\mathbf{E}}\left[\xi(x) h^{\prime}(x)\right]}{\sqrt{n}}+\frac{\overline{\mathbf{E}}\left[\xi^{2}(x) h^{\prime \prime}(x)\right]}{2 n}+\overline{\mathbf{E}} \delta\left(x, \frac{\xi(x)}{\sqrt{n}} \mid h\right) .
$$


We are going to estimate values $\overline{\mathbf{E}}\left[\xi(x) h^{\prime}(x)\right]$ and $\overline{\mathbf{E}}\left[\xi^{2}(x) h^{\prime \prime}(x)\right]$. To simplify notations, we will write in the proof $\alpha(x)$ and $\beta(x)$ instead of $\alpha_{\varkappa}(x \mid h)$ and $\beta_{\varkappa, \kappa}(x \mid h)$. We will use the following property

$$
\overline{\mathbf{E}}[c(X-a)]=\left\{\begin{array}{l}
c(\overline{\mathbf{E}}[X]-a)=|c|(\overline{\mathbf{E}}[X]-a), \text { if } c \geq 0, \\
|c|(a+\overline{\mathbf{E}}[-X])=|c|(a-\underline{\mathbf{E}}[X]), \text { if } c \leq 0,
\end{array}\right.
$$

which takes place for all constants $a, c$ and any variable $X \in \mathcal{H}$ in a sublinear expectation space $(\Omega, \mathcal{H}, \overline{\mathbf{E}})$.

First, if $h^{\prime}(x) \geq 0$ then, by (48)

$$
\overline{\mathbf{E}}\left[\left(X_{0}-\alpha(x)\right) h^{\prime}(x)\right]=\left|h^{\prime}(x)\right|(\bar{\mu}-\alpha(x)) .
$$

But condition $\alpha(x) \neq \bar{\mu}$ when $h^{\prime}(x) \geq 0$ implies that $\left|h^{\prime}(x)\right| \leq \varkappa$ and $\alpha(x) \geq$ $(\bar{\mu}+\underline{\mu}) / 2$. Hence

$$
\overline{\mathbf{E}}\left[\left(X_{0}-\alpha(x)\right) h^{\prime}(x)\right] \leq \varkappa(\bar{\mu}-\alpha(x)) \leq \varkappa(\bar{\mu}-\underline{\mu}) / 2, \quad \text { when } h^{\prime}(x) \geq 0 .
$$

Similarly, when $h^{\prime}(x)<0$, using again (48), we obtain that

$$
\overline{\mathbf{E}}\left[\left(X_{0}-\alpha(x)\right) h^{\prime}(x)\right]=\left|h^{\prime}(x)\right|\left(\alpha(x)-\underline{\mathbf{E}}\left[X_{0}\right]\right)=\left|h^{\prime}(x)\right|(\alpha(x)-\underline{\mu}) .
$$

But by (36) and (37), condition $\alpha(x) \neq \underline{\mu}$ when $h^{\prime}(x)<0$ implies that $\left|h^{\prime}(x)\right| \leq$ $\varkappa$ and $\alpha(x) \leq(\bar{\mu}+\underline{\mu}) / 2$. Hence

$$
\overline{\mathbf{E}}\left[\left(X_{0}-\alpha(x)\right) h^{\prime}(x)\right] \leq \varkappa(\alpha(x)-\underline{\mu}) \leq \varkappa(\bar{\mu}-\underline{\mu}) / 2, \quad \text { when } h^{\prime}(x)<0 .
$$

Thus, for all values of $h^{\prime}(x)$ we have

$$
\overline{\mathbf{E}}\left[\xi(x) h^{\prime}(x)\right]=\frac{1}{\beta(x)} \overline{\mathbf{E}}\left[\left(X_{0}-\alpha(x)\right) h^{\prime}(x)\right] \leq \frac{\varkappa(\bar{\mu}-\underline{\mu})}{2 \underline{\sigma}} .
$$

Next, if $h^{\prime \prime}(x) \geq 0$ then, by (41),

$$
\overline{\mathbf{E}}\left[h^{\prime \prime}(x) \xi^{2}(x)\right]=h^{\prime \prime}(x) \overline{\mathbf{E}}\left[\frac{\left(X_{0}-\alpha(x)\right)^{2}}{\beta^{2}(x)}\right]=h^{\prime \prime}(x) \frac{\bar{\sigma}^{2}(\alpha(x))}{\beta^{2}(x)}
$$

But by (39) and (38), condition $\beta^{2}(x) \neq \bar{\sigma}^{2}(\alpha(x))$ when $h^{\prime \prime}(x) \geq 0$ implies that $h^{\prime \prime}(x) \leq \kappa$. Hence

$$
\overline{\mathbf{E}}\left[h^{\prime \prime}(x) \xi^{2}(x)\right]-h^{\prime \prime}(x)=h^{\prime \prime}(x)\left(\frac{\bar{\sigma}^{2}(\alpha(x))}{\beta^{2}(x)}-1\right) \leq \kappa\left(\frac{\bar{\sigma}^{2}}{\underline{\sigma}^{2}}-1\right), \quad \text { if } \quad h^{\prime \prime}(x) \geq 0 .
$$

At last, when $h^{\prime \prime}(x)<0$, we have from (41) and (48) that

$$
\begin{array}{r}
\overline{\mathbf{E}}\left[h^{\prime \prime}(x) \xi^{2}(x)\right]-h^{\prime \prime}(x)=\left|h^{\prime \prime}(x)\right|\left(-\underline{\mathbf{E}}\left[\xi^{2}(x)\right]+1\right) \\
=\left|h^{\prime \prime}(x)\right|\left(1-\underline{\mathbf{E}}\left[\frac{\left(X_{0}-\alpha(x)\right)^{2}}{\beta^{2}(x)}\right]\right)=\left|h^{\prime \prime}(x)\right|\left(1-\frac{\underline{\sigma}^{2}(\alpha(x))}{\beta^{2}(x)}\right) .
\end{array}
$$


By (39) and (38), condition $\beta^{2}(x) \neq \underline{\sigma}^{2}(\alpha(x))$ when $h^{\prime \prime}(x)<0$ implies that $h^{\prime \prime}(x) \leq \kappa$. Hence

$$
\overline{\mathbf{E}}\left[h^{\prime \prime}(x) \xi^{2}(x)\right]-h^{\prime \prime}(x) \leq \kappa\left(1-\frac{\underline{\sigma}^{2}(\alpha(x))}{\beta^{2}(x)}\right) \leq \kappa\left(1-\frac{\sigma^{2}}{\bar{\sigma}^{2}}\right), \text { when } h^{\prime \prime}(x)<0 .
$$

Note that in (50) and (51) we also used (40). But $1-\underline{\sigma}^{2} / \bar{\sigma}^{2} \leq \bar{\sigma}^{2} / \underline{\sigma}^{2}-1$. Thus for all values of $h^{\prime \prime}(x)$

$$
\overline{\mathbf{E}}\left[h^{\prime \prime}(x) \xi^{2}(x)\right]-h^{\prime \prime}(x) \leq \kappa\left(\bar{\sigma}^{2} / \underline{\sigma}^{2}-1\right) .
$$

Substituting now (49) and (52) into (47), we obtain that

(53) $\overline{\mathbf{E}} h\left(x+\frac{\xi(x)}{\sqrt{n}}\right) \leq h(x)+\frac{\varkappa(\bar{\mu}-\underline{\mu})}{2 \sqrt{n} \underline{\sigma}}+\frac{\kappa}{2 n}\left(\frac{\bar{\sigma}^{2}}{\underline{\sigma}^{2}}-1\right)+\overline{\mathbf{E}} \delta\left(x, \frac{\xi(x)}{\sqrt{n}} \mid h\right)$.

So, the desired inequality (42) follows from (46) and (53).

3.3. Smoothing. Introduce functions

$$
g_{r}(x)=\frac{2\left((r-|x|)^{+}\right)^{2}-\left((r-2|x|)^{+}\right)^{2}}{r^{3}}, \quad x \in \mathbb{R}, \quad r>0 .
$$

Lemma 2. For each $r>0$ function $g_{r}(x)$ is a continuous density on $\mathbb{R}$ with a derivative $g_{r}^{\prime}(x)$ which is absolutely continuous on $\mathbb{R}$. It's second derivative $g_{r}^{\prime \prime}(x)$ is also continuous for all $x \in \mathbb{R}$, but $x= \pm r$ and $x= \pm r / 2$. Moreover,

$$
\bar{\varepsilon}_{r}(y):=\frac{1}{2} \int\left|\varepsilon_{r}(x, y)\right| d x \leq 16 \min \left\{\frac{|y|^{3}}{(2 r)^{3}}, \frac{y^{2}}{(2 r)^{2}}\right\} \leq 16 \min \left\{\frac{|y|^{p}}{(2 r)^{p}}, \frac{y^{2}}{(2 r)^{2}}\right\},
$$

for all $r>0, p \in[2,3]$ and $y \in \mathbb{R}$, where

$$
\varepsilon_{r}(x, y):=g_{r}(x-y)-g_{r}(x)+y g_{r}^{\prime}(x)-y^{2} g_{r}^{\prime \prime}(x) / 2 .
$$

Proof. If $r=1 / 2$ then the assertion follows from the proof of Lemma 6.2 in Sakhanenko (2000). The case of arbitrary $r>0$ follows from the evident representations:

$$
g_{r}(x)=\frac{1}{2 r} g_{1 / 2}\left(\frac{x}{2 r}\right), \quad \varepsilon_{r}(x, y)=\frac{1}{2 r} \varepsilon_{1 / 2}\left(\frac{x}{2 r}, \frac{y}{2 r}\right), \quad \bar{\varepsilon}_{r}(y)=\bar{\varepsilon}_{1 / 2}\left(\frac{y}{2 r}\right) .
$$

Suppose now that for each $\vec{x} \in R^{m}$ we are given a measurable set $A(\vec{x}) \subset \mathbb{R}$.

Lemma 3. Let random vector $\vec{\eta}$ be independent from random variable $\nu$ with density $g_{1}(\cdot)$. Then the following function

$$
h(x):=\mathbf{P}(x+r \nu \in A(\vec{\eta})), \quad r>0,
$$

is twice continuously differentiable for all $x \in \mathbb{R}$ and

$$
\bar{\delta}(y \mid h):=\sup _{x \in \mathbb{R}}|\delta(x, y \mid h)| \leq \bar{\varepsilon}_{r}(y)
$$

for each $r>0$ and $y \in \mathbb{R}$, where the function $\delta(x, y \mid h)$ was defined in 4.3). 
Proof. Remind that for each non-random $\vec{s} \in \mathbb{R}^{m}$

$$
\mathbf{P}(x+r \nu \in A(\vec{s}))=\int_{A(\vec{s})} g_{r}(z-x) d z,
$$

where $g_{r}(\cdot)$ is the density of $r \nu$. Now for any $\vec{\eta}$ independent from $\nu$ we have

$$
h(x)=\mathbf{P}(x+r \nu \in A(\vec{\eta}))=\mathbf{E} \int_{A(\vec{\eta})} g_{r}(z-x) d z
$$

From definitions (43), (56) and (58) it is not difficult to obtain that

$$
\delta(x, y \mid h)=\mathbf{E} \int_{A(\vec{\eta})} \varepsilon_{r}(z-x, y) d z .
$$

Note that

$$
\int \varepsilon_{r}(x, y) d x=0=\int \varepsilon_{r}^{+}(x, y) d x-\int \varepsilon_{r}^{-}(x, y) d x .
$$

Hence, we have from (59) that

$$
\begin{aligned}
& \delta(x, y \mid h) \leq \int \varepsilon_{r}^{+}(z-x, y) d z=\int \varepsilon_{r}^{+}(x, y) d x=\frac{1}{2} \int\left|\varepsilon_{r}(x, y) d x\right| d x, \\
& -\delta(x, y \mid h) \leq \int \varepsilon_{r}^{-}(z-x, y) d z=\int \varepsilon_{r}^{-}(x, y) d x=\frac{1}{2} \int\left|\varepsilon_{r}(x, y) d x\right| d x .
\end{aligned}
$$

These two inequalities imply (57).

Lemma 4. Let random vector $\vec{\eta}$ be independent from random variable $Z \sim$ $N(0,1)$. Then the following function

$$
h(x):=\mathbf{P}(x+b Z \in A(\vec{\eta})), \quad b \neq 0,
$$

is twice continuously differentiable for all $x \in \mathbb{R}$ and

$$
\bar{\delta}(y \mid h):=\sup _{x \in \mathbb{R}}|\delta(x, y \mid h)| \leq 0.4|y / b|^{3}
$$

for each $y \in \mathbb{R}$, where the function $\delta(x, y \mid h)$ was defined in 43.).

Proof. Similarly to the proof of Lemma 3 we have

$$
h(x)=\mathbf{P}(x+b Z \in A(\vec{\eta}))=\mathbf{E} \int_{A(\vec{\eta})} p_{b}(z-x) d z
$$

where $p_{b}(\cdot)$ is the density of $b Z$. Now introduce

$$
\varepsilon(x, y):=p_{b}(x-y)-p_{b}(x)+y p_{b}^{\prime}(x)-\frac{y^{2} p_{b}^{\prime \prime}(x)}{2}=\frac{y^{3}}{2} \int_{0}^{1}(1-t)^{2} p_{b}^{\prime \prime \prime}(x-t y) d t .
$$

From definitions (43), (61) and (62) it is not difficult to obtain that

$$
\delta(x, y \mid h)=\mathbf{E} \int_{A(\vec{\eta})} \varepsilon(z-x, y) d z=\frac{y^{3}}{2} \int_{0}^{1}(1-t)^{2} \mathbf{E} \int_{A(\vec{\eta})} p_{b}^{\prime \prime \prime}(z-x-t y) d z d t .
$$


So, we have that

$$
\begin{aligned}
& |\delta(x, y \mid h)| \leq \frac{|y|^{3}}{2} \int_{0}^{1}(1-t)^{2} \int\left|p_{b}^{\prime \prime \prime}(z-x-t y)\right| d z d t \\
= & \frac{|y|^{3}}{2} \int_{0}^{1}(1-t)^{2} d t \cdot \int\left|p_{b}^{\prime \prime \prime}(y)\right| d y=\frac{|y|^{3}}{6|b|^{3}} \int\left|p_{1}^{\prime \prime \prime}(x)\right| d x .
\end{aligned}
$$

It is easy to see that

$$
p_{1}^{\prime}(x)=-x p_{1}(x), \quad p_{1}^{\prime \prime}(x)=\left(x^{2}-1\right) p_{1}(x) \quad \text { and } \quad p_{1}^{\prime \prime \prime}(x)=\left(3 x-x^{3}\right) p_{1}(x) .
$$

Since $\left|p_{1}^{\prime \prime \prime}(x)\right|$ is even and $\int p_{1}^{\prime \prime \prime}(x) d x=0$, we obtain

$$
\begin{gathered}
\int\left|p_{1}^{\prime \prime \prime}(x)\right| d x=2 \int\left(p_{1}^{\prime \prime \prime}(x)\right)^{+} d x=4 \int_{0}^{\infty}\left(p_{1}^{\prime \prime \prime}(x)\right)^{+} d x=4 \int_{0}^{\sqrt{3}}\left(3 x-x^{3}\right) p_{1}(x) d x \\
=4 \int_{0}^{\sqrt{3}} p_{1}^{\prime \prime \prime}(x) d x=4 p_{1}^{\prime \prime}(\sqrt{3})-4 p_{1}^{\prime \prime}(0)=\frac{4}{\sqrt{2 \pi}}\left(1+\frac{2}{e^{3 / 2}}\right)<2.4 .
\end{gathered}
$$

Thus, substituting this fact into (63), we obtain the desired inequality (60).

\section{Proofs of Theorem 1 and it's corollaries}

In all cases when we are given $n$ values, say $x_{1}, \ldots, x_{n}$, denoted by the same letter with indices, we agree to use everywhere vector notation: $\vec{x}_{k}=\left(x_{1}, \ldots, x_{k}\right)$, for $k=1, \ldots, n$.

\subsection{Representations for broken lines. Define}

$$
\zeta_{i}:=\left(X_{i}-\mu_{i}\right) /\left(\sqrt{n} \sigma_{i}\right), \quad i=1,2, \ldots, n,
$$

where the values $\mu_{i}$ and $\sigma_{i}$ are continuous functions of $\vec{X}_{i-1}=\left(X_{1}, X_{2}, \ldots, X_{i-1}\right)$ which will be specified later. Then the random broken line $S_{n}$ with the values (23) at key points can be rewritten as

$$
S_{n}(t)=\sum_{i=1}^{n} \zeta_{i} e_{i}(t), \quad t \in[0,1],
$$

where growing functions $e_{i}$ have the following form:

$$
e_{i}(t)=e_{i, n}(t):=\left\{\begin{array}{l}
0, \text { for } t \leq \frac{i-1}{n}, \\
n t-(i-1), \text { for } t \in\left[\frac{i-1}{n}, \frac{i}{n}\right], \\
1, \text { for } t \geq \frac{i}{n} .
\end{array}\right.
$$

Here $i=0,1, \ldots, n+1$. Note that $e_{0, n}(t)=1$ and $e_{n+1, n}(t)=0$ for all $t \in[0,1]$.

Let $B=B(\cdot)$ be a standard Wiener process, i.e., $\mathbf{E} B(t)=0$ and $\mathbf{E} B^{2}(t)=t$ for all $t \in[0,1]$. Then for each $n=1,2, \ldots$ and all $t \in[0,1]$ define

$$
B_{n}(t):=\sum_{i=1}^{n} \theta_{i} e_{i}(t) \quad \text { with } \quad \theta_{i}:=\theta_{i, n}=B\left(\frac{i}{n}\right)-B\left(\frac{i-1}{n}\right) .
$$


Thus, $B_{n}=B_{n}(\cdot)$ is also a random broken line.

4.2. Main idea. We are going to describe a method how it is possible to change in (64) variables $\zeta_{n}, \zeta_{n-1}, \ldots, \zeta_{2}, \zeta_{1}$ one by one into $\theta_{n}, \theta_{n-1}, \ldots, \theta_{2}, \theta_{1}$, and to obtain (28) as a result. Fix an integer $n \geq 1$, real numbers $\rho, r>0$ and an arbitrary set $D \subset \mathbb{C}[0,1]$. Introduce a sequence $\nu, \nu_{1}, \nu_{2}, \ldots$ of i.i.d. classical random variables with density $g_{1}(\cdot)$ from (54), which we suppose to be independent from the Wiener process $B$. Underline that $|\nu|<1$ and $\left|\nu_{i}\right|<1$ a.s. for all $i$. We have in mind that almost surely

$$
|r \nu|<r \quad \text { and } \quad\left\|\sum_{i=1}^{n} \frac{\rho}{n} \nu_{i} e_{i}\right\|=\max _{t \in[0,1]}\left|\sum_{i=1}^{n} \frac{\rho}{n} \nu_{i} e_{i}(t)\right| \leq \sum_{i=1}^{n} \frac{\rho}{n}=\rho
$$

for some negligible $\rho>0$ which will be chosen later. We have from (66) that

$$
h_{n+1}\left(\vec{x}_{n}\right):=\mathbf{P}\left(\sum_{i=1}^{n}\left(x_{i}+\frac{\rho}{n} \nu_{i}\right) e_{i} \in D^{\rho}\right) \geq \mathbf{I}\left(\sum_{i=1}^{n} x_{i} e_{i} \in D\right) .
$$

In the next subsection we construct a special sequence of sets $D_{k}$ which have, among others, the following property

$$
D^{\rho}=D_{n+1} \subset \ldots \subset D_{k+1} \subset D_{k} \subset \ldots \subset D_{0} \subset D^{\rho+r}, \quad 1 \leq k \leq n .
$$

In particular, it follows from (66) and (68) that

$$
h_{0}:=\mathbf{P}\left(B_{n}+r \nu e_{0}+\sum_{i=1}^{n} \frac{\rho}{n} \nu_{i} e_{i} \in D_{0}\right) \leq \mathbf{P}\left(B_{n} \in D^{2 \rho+2 r}\right) .
$$

Definition (22) and (67) imply that $\overline{\mathbf{P}}\left(S_{n} \in D\right) \leq \overline{\mathbf{E}} h_{n+1}\left(\vec{\zeta}_{n}\right)$. Using also (69) we obtain:

$$
\Delta:=\overline{\mathbf{P}}\left(S_{n} \in D\right)-\mathbf{P}\left(B_{n} \in D^{2 \rho+2 r}\right) \leq \overline{\mathbf{E}} h_{n+1}\left(\vec{\zeta}_{n}\right)-h_{0} .
$$

Thus, for any continuous and bounded functions $h_{1}, \ldots, h_{n}$

$$
\Delta \leq \sum_{k=1}^{n+1} \Delta_{k} \quad \text { with } \quad \Delta_{k}=\overline{\mathbf{E}} h_{k}\left(\vec{\zeta}_{k}\right)-\overline{\mathbf{E}} h_{k-1}\left(\vec{\zeta}_{k-1}\right) .
$$

Values $\Delta_{k}$ will be estimated in subsection 4.6 with $h_{k}$ from (72) below, and with special $\mu_{k}$ and $\sigma_{k}$ which will be constructed in subsection 4.5 .

4.3. Construction of sets $D_{k}$. Similar to Sakhanenko [9] and [11, we introduce a sequence of sets $D_{k}$ which we will interpret as the sequence of enlargements of the set $D$. We first define $D_{n+1}=D^{\rho}$. After that for each $k=n, n-1, \ldots, 2,1,0$ we introduce sets $D_{k}$ by the following rule:

$$
D_{k}=D_{k}(r, \rho):=\left\{\tilde{x}=\tilde{y}+z_{k}\left(e_{k}-e_{k+1}\right) \in \mathbb{C}[0,1]: \tilde{y} \in D_{k+1},\left|z_{k}\right|<r\right\} .
$$

Now for $k=1, \ldots, n$ introduce functions

$$
h_{k}\left(\vec{x}_{k}\right):=\mathbf{P}\left(\sum_{i=1}^{k}\left(x_{i}+\frac{\rho}{n} \nu_{i}\right) e_{i}+r \nu e_{k}+\sum_{j=k+1}^{n}\left(\theta_{j}+\frac{\rho}{n} \nu_{j}\right) e_{j} \in D_{k}\right),
$$


where we assume that $\sum_{\emptyset}=0$. Since $e_{n}=e_{n}-e_{n+1}$, we have from definitions (67) and (711) of $D_{n+1}$ and $D_{n}$ that

$$
h_{n+1}\left(\vec{x}_{n}\right) \leq h_{n}\left(\vec{x}_{n}\right)=\mathbf{P}\left(r \nu e_{n}+\sum_{i=1}^{n}\left(x_{i}+\frac{\rho}{n} \nu_{i}\right) e_{i} \in D_{n}\right) ; \quad \text { so that } \quad \Delta_{n+1} \leq 0 .
$$

Lemma 5. For each $r>0$ and $\rho>0$ inclusion of sets (68) takes place for sets $D_{k}$ defined in (71). Moreover for $k=1, \ldots, n$

$$
\mathbf{E} h_{k}\left(\vec{x}_{k-1}, \theta_{k}\right) \leq h_{k-1}\left(\vec{x}_{k-1}\right) .
$$

Proof. From the definition (171) we have

$$
D_{0}=\left\{\tilde{x}=\tilde{y}+\tilde{z}: \tilde{y} \in D^{\rho}, \tilde{z}=\sum_{k=0}^{n} z_{k}\left(e_{k}-e_{k+1}\right), \max \left|z_{k}\right|<r\right\} .
$$

In particular,

$$
\|\tilde{z}\|=\max _{t \in[0,1]}|\tilde{z}(t)| \leq r \max _{t \in[0,1]} \sum_{k=0}^{n}\left|e_{k}(t)-e_{k+1}(t)\right| .
$$

But $e_{k}(t)=e_{0}(t-k / n)$ for all $t$ as it follows from definition (65) of nondecreasing functions $e_{k}$. Hence

$\sum_{k=0}^{n}\left|e_{k}(t)-e_{k+1}(t)\right|=\sum_{k=0}^{n}\left[e_{0}\left(t-\frac{k}{n}\right)-e_{0}\left(t-\frac{k+1}{n}\right)\right]=e_{0}(t)-e_{0}\left(t-\frac{n+1}{n}\right) \leq 1$.

Thus $\|\tilde{z}\|<r$ and we have from (5) that $D_{0} \subset D^{\rho+r}$.

All other inclusions in (68) follow immediately from definitions (71).

Now we are going to prove the main property (74) of sets $D_{k}$ introduced in (71). From definition (72) we have:

$$
\mathbf{E} h_{k}\left(\vec{x}_{k-1}, \theta_{k}\right)=\mathbf{P}\left(\sum_{i=1}^{k-1}\left(x_{i}+\frac{\rho}{n} \nu_{i}\right) e_{i}+r \nu e_{k}+\sum_{j=k}^{n}\left(\theta_{j}+\frac{\rho}{n} \nu_{j}\right) e_{j} \in D_{k}\right) .
$$

But if $\tilde{x}+r \nu e_{k} \in D_{k}$ for some $\tilde{x} \in \mathbb{C}[0,1]$, then it follows from the definition (71) of $D_{k-1}$ with $z_{k-1}=r \nu$ that

$$
\tilde{x}+r \nu e_{k-1}=\tilde{x}+r \nu e_{k}+z_{k-1}\left(e_{k-1}-e_{k}\right) \in D_{k-1} .
$$

This fact and (75) together with (72) imply that

$$
\mathbf{E} h_{k}\left(\vec{x}_{k-1}, \theta_{k}\right)=\mathbf{P}\left(\tilde{x}+r \nu e_{k} \in D_{k}\right) \leq \mathbf{P}\left(\tilde{x}+r \nu e_{k-1} \in D_{k-1}\right)=h_{k-1}\left(\vec{x}_{k-1}\right)
$$

with evident random $\tilde{x}$. Thus, (74) is proved.

4.4. Using Taylor formula. All broken lines under probability in (72) has summand $\sum_{i=1}^{n} \frac{\rho}{n} \nu_{i} e_{i}(t)$. We are going to show that it makes all functions $h_{k}$ to be sufficiently smooth. 
Lemma 6. For each $k=1, \ldots, n$ function $h_{k}\left(\vec{x}_{k}\right)=h_{k}\left(\vec{x}_{k-1}, x_{k}\right)$ is twice continuously differentiable with respect to its last variable $x_{k}$. In addition, the following functions

$$
h_{k}^{\prime}\left(\vec{x}_{k-1}, 0\right)=\left.\frac{\partial}{\partial x} h_{k}\left(\vec{x}_{k-1}, x\right)\right|_{x=0} \quad \text { and } \quad h_{k}^{\prime \prime}\left(\vec{x}_{k-1}, 0\right)=\left.\frac{\partial^{2}}{\partial x^{2}} h_{k}\left(\vec{x}_{k-1}, x\right)\right|_{x=0}
$$

are uniformly continuous functions of $\vec{x}_{k-1}$.

Proof. Consider the set $D_{k}^{*}=\left\{\vec{x}_{n}=\left(\vec{x}_{k-1}, x_{k}, \vec{x}_{k, n}\right): \sum_{i=1}^{n} x_{i} e_{i} \in D_{k}\right\}$. It is easy to see that

$$
\begin{aligned}
& h_{k}\left(\vec{x}_{k}\right)=P\left(\left(\vec{x}_{k-1}+\frac{\rho}{n} \vec{\nu}_{k-1}, x_{k}+r \nu+\frac{\rho}{n} \nu_{k}, \vec{\theta}_{k, n}+\frac{\rho}{n} \vec{\nu}_{k, n}\right) \in D_{k}^{*}\right) \\
& =\int d z \underbrace{\int \ldots \int_{i=1}^{k-1}}_{D_{k}^{*}} g\left(y_{i}-x_{i}\right) g\left(y_{k}\right) g_{r}\left(z-x_{k}-y_{k}\right) \tilde{g}\left(\vec{y}_{k, n}\right) d y_{1} \ldots d y_{n},
\end{aligned}
$$

where $g(\cdot)=g_{\rho / n}(\cdot)$ is the density of $\frac{\rho}{n} \nu_{i}$ for all $i, g_{r}(\cdot)$ is the density of $r \nu$ and $\tilde{g}(\cdot)$ is the joint density of $\vec{\theta}_{k, n}+\frac{\rho}{n} \vec{\nu}_{k, n}$. Using (77), it is not difficult to verify that for all $l<k$

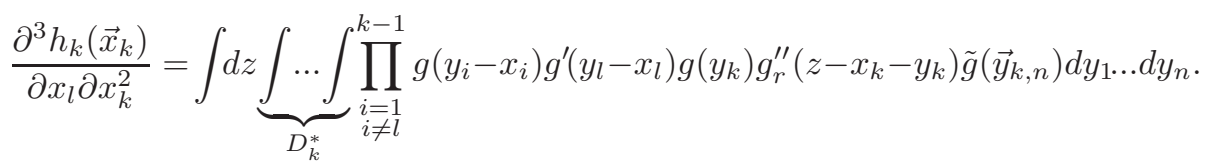

Thus, with $z_{i}=y_{i}-x_{i}$ and $z_{k}=z-x_{k}-y_{k}$ we obtain that

$$
\begin{aligned}
& \left|\frac{\partial^{3} h_{k}\left(\vec{x}_{k}\right)}{\partial x_{l} \partial x_{k}^{2}}\right| \leq \iiint\left|g^{\prime}\left(y_{l}-x_{l}\right) g_{r}^{\prime \prime}\left(z-x_{k}-y_{k}\right)\right| g\left(y_{k}\right) d y_{l} d z d y_{k} \\
= & \int\left|g_{r}^{\prime \prime}\left(z_{k}\right)\right| d z_{k} \cdot \int\left|g^{\prime}\left(z_{l}\right)\right| d z_{l}=\frac{1}{r^{2}} \int\left|g_{1}^{\prime \prime}(x)\right| d x \cdot \frac{n}{\rho} \int\left|g_{1}^{\prime}(x)\right| d x<\infty .
\end{aligned}
$$

So, from (78) we have that $h_{k}^{\prime \prime}\left(\vec{x}_{k-1}, 0\right)$ is uniformly continuous because all its partial derivatives are uniformly bounded. The similar arguments are true also for function $h_{k}\left(\vec{x}_{k}\right)$ and for $h_{k}^{\prime}\left(\vec{x}_{k-1}, 0\right)$ from (76).

Introduce an error term of the Taylor expansion of function $h_{k}\left(\vec{x}_{k-1}, x_{k}\right)$ with respect to its last variable $x_{k}=x$ at $x_{k}=x=0$ :

$$
\delta_{k}\left(y \mid \vec{x}_{k-1}\right)=\delta(0, y \mid h) \quad \text { for } \quad h(\cdot)=h_{k}\left(\vec{x}_{k-1}, \cdot\right), \quad k=1, \ldots, n,
$$

where the function $\delta(x, y \mid h)$ was defined in (43).

Lemma 7. If $p \in[2,3]$, then for each $k=1, \ldots, n$ and all $y \in \mathbb{R}$

$$
\delta_{k}(y):=\sup _{\vec{x}_{k-1}}\left|\delta_{k}\left(y \mid \vec{x}_{k-1}\right)\right| \leq \bar{\varepsilon}_{p, r}(y):=16 \min \left\{\frac{|y|^{p}}{(2 r)^{p}}, \frac{y^{2}}{(2 r)^{2}}\right\} .
$$


Proof. Introduce set:

$$
\left.A_{k}\left(\vec{y}_{k-1}, y, \vec{y}_{k, n}\right)\right)=\left\{x: \sum_{i=1}^{k-1} y_{i} e_{i}+(x+y) e_{k}+\sum_{j=k+1}^{n} y_{j} e_{j} \in D^{k}\right\} .
$$

Then from (72) we have

$$
h(x)=h_{k}\left(\vec{x}_{k-1}, x\right)=\mathbf{P}\left(x+r \nu \in A_{k}\left(\vec{x}_{k-1}+\frac{\rho}{n} \vec{\nu}_{k-1}, \frac{\rho}{n} \nu_{k}, \vec{\theta}_{k, n}+\frac{\rho}{n} \vec{\nu}_{k, n}\right)\right) .
$$

Applying Lemma 3 to the random set $\left.A_{k}\left(\vec{x}_{k-1}+\frac{\rho}{n} \vec{\nu}_{k-1}, \frac{\rho}{n} \nu_{k}, \vec{\theta}_{k, n}+\frac{\rho}{n} \vec{\nu}_{k, n}\right)\right)$, we obtain from (57) that $\delta_{k}(y) \leq \bar{\varepsilon}_{r}(y)$ with a function $\bar{\varepsilon}_{r}(y) \leq \bar{\varepsilon}_{p, r}(y)$ by (55)).

4.5. Constructions of $\mu_{k}$ and $\sigma_{k}$. Choose numbers $\varkappa>0$ and $\kappa>0$ so that

$$
\frac{\varkappa(\bar{\mu}-\underline{\mu})}{2 \sqrt{n} \underline{\sigma}}+\frac{\kappa}{2 n}\left(\frac{\bar{\sigma}^{2}}{\underline{\sigma}^{2}}-1\right) \leq \rho_{0} \min _{k \leq n} \mathbf{E}\left[\delta_{k}\left(\frac{Z}{\sqrt{n}}\right)\right], \quad \rho_{0}=0.001,
$$

where $Z \sim N(0,1)$. After that define functions

$$
\alpha_{k}\left(\vec{x}_{k-1}\right):=\alpha_{\varkappa}(0 \mid h) \quad \text { for } \quad h(\cdot)=h_{k}\left(\vec{x}_{k-1}, \cdot\right), \quad k=2, \ldots, n,
$$

where function $\alpha_{\varkappa}(0 \mid h)$ was defined in (36) and (37); and we put

$$
\beta_{k}\left(\vec{x}_{k-1}\right)=\beta_{\varkappa, \kappa}(0 \mid h) \quad \text { for } \quad h(\cdot)=h_{k}\left(\vec{x}_{k-1}, \cdot\right), \quad k=2, \ldots, n
$$

with function $\beta_{\varkappa, \kappa}(0 \mid h)$ introduced in (38) and (39).

Lemma 8. For each $k=2, \ldots, n$ functions $\alpha_{k}\left(\vec{x}_{k-1}\right)$ and $\beta_{k}\left(\vec{x}_{k-1}\right)$ are continuous functions of $\vec{x}_{k-1} \in \mathbb{R}^{k-1}$ and satisfy properties 40]. Moreover, for all $\vec{x}_{k-1} \in \mathbb{R}^{k-1}$

$$
H_{k}\left(\vec{x}_{k-1}\right):=\overline{\mathbf{E}} h_{k}\left(\vec{x}_{k-1}, \xi_{k}\left(\vec{x}_{k-1}\right) / \sqrt{n}\right) \leq h_{k-1}\left(\vec{x}_{k-1}\right)+\tau_{k, n},
$$

where $\xi_{k}\left(\vec{x}_{k-1}\right):=\left(X_{0}-\alpha_{k}\left(\vec{x}_{k-1}\right)\right) / \beta_{k}\left(\vec{x}_{k-1}\right)$ and

$$
\tau_{k, n}=\sup _{\mu \in[\underline{\mu}, \bar{\mu}]} \overline{\mathbf{E}}\left[\delta_{k}\left(\xi_{0}(\mu) / \sqrt{n}\right)\right]+\left(1+\rho_{0}\right) \mathbf{E}\left[\delta_{k}(Z / \sqrt{n})\right] .
$$

Proof. We are going to apply Lemma 1 at $x=0$ to auxiliary function $h(\cdot)$ used in (79), (82) and (83). Note that in this case $\xi(0)=\xi_{k}\left(\vec{x}_{k-1}\right)$ and $Z=\sqrt{n} \theta_{k}$ has standard normal distribution. Hence, using (80) and (81) we obtain from (42) with $x=0$ that

$$
\overline{\mathbf{E}} h_{k}\left(\vec{x}_{k-1}, \frac{\xi_{k}\left(\vec{x}_{k-1}\right)}{\sqrt{n}}\right) \leq \mathbf{E} h_{k}\left(\vec{x}_{k-1}, \theta_{k}\right)+\overline{\mathbf{E}} \delta_{k}\left(\frac{\xi_{k}\left(\vec{x}_{k-1}\right)}{\sqrt{n}}\right)+\left(1+\rho_{0}\right) \mathbf{E} \delta_{k}\left(\theta_{k}\right) .
$$

Note that $\left|\xi_{k}\left(\vec{x}_{k-1}\right)\right| \leq\left|\xi_{0}(\mu)\right|$ with $\mu=\alpha_{k}\left(\vec{x}_{k-1}\right)$ as it follows from (40). This fact together with (866) yields

$$
H_{k}\left(\vec{x}_{k-1}\right)=\overline{\mathbf{E}} h_{k}\left(\vec{x}_{k-1}, \xi_{k}\left(\vec{x}_{k-1}\right) / \sqrt{n}\right) \leq \mathbf{E} h_{k}\left(\vec{x}_{k-1}, \theta_{k}\right)+\tau_{k, n}
$$

Here we used also definitions from (84) and (85).

Therefore (84) follows from (87) and (74). 
Now for $k=1,2, \ldots, n$ we are going to define functions $\mu_{k}\left(\vec{x}_{k-1}\right)$ and $\sigma_{k}\left(\vec{x}_{k-1}\right)$. We first introduce

$$
\mu_{1}=\mu_{1}\left(\vec{x}_{0}\right)=\underline{\mu} \text { and } \sigma_{1}=\sigma_{1}\left(\vec{x}_{0}\right)=\underline{\sigma}(\underline{\mu})>0 .
$$

Note that if for some $k>1$ all $\mu_{i}$ and $\sigma_{i}$ for $i<k$ are defined, then we may put

$$
z_{i}\left(\vec{x}_{i}\right)=\left(x_{i}-\mu_{i}\left(\vec{x}_{i-1}\right)\right) / \sqrt{n} \sigma_{i}\left(\vec{x}_{i-1}\right), \quad i=1,2, \ldots, k,
$$

and define vector $\vec{z}_{k-1}\left(\vec{x}_{k-1}\right)=\left(z_{1}\left(\vec{x}_{1}\right), \ldots, z_{k-1}\left(\vec{x}_{k-1}\right)\right)$. At last, after that we may introduce

(89) $\mu_{k}\left(\vec{x}_{k-1}\right)=\alpha_{k}\left(\vec{z}_{k-1}\left(\vec{x}_{k-1}\right)\right), \quad \sigma_{k}\left(\vec{x}_{k-1}\right)=\beta_{k}\left(\vec{z}_{k-1}\left(\vec{x}_{k-1}\right)\right), \quad k=2, \ldots, n$.

So, later on we use in our proof only $\zeta_{k}=z_{k}\left(\vec{X}_{k}\right)=\left(X_{k}-\mu_{k}\right) / \sqrt{n} \sigma_{k}$ with $\mu_{k}=\mu_{k}\left(\vec{X}_{k-1}\right)$ and $\sigma_{k}=\sigma_{k}\left(\vec{X}_{k-1}\right)$ defined in (88) and (89).

Lemma 9. If all values $\mu_{k}$ and $\sigma_{k}$ are defined in (88) and (89), then

$$
\Delta:=\overline{\mathbf{P}}\left(S_{n} \in D\right)-\mathbf{P}\left(B_{n} \in D^{2 \rho+2 r}\right) \leq \sum_{k=1}^{n} \tau_{k, n} .
$$

In addition, for all $k=1, \ldots, n$

$$
\tau_{k, n} \leq 16\left\{\frac{\gamma_{p}(2 r \sqrt{n})}{(2 r \sqrt{n})^{p}}+\left(1+\rho_{0}\right) \mathbf{E} \min \left\{\frac{|Z|^{p}}{(2 r \sqrt{n})^{p}}, \frac{Z^{2}}{(2 r \sqrt{n})^{2}}\right\}\right\}
$$

Proof. Introduce function:

(92) $\bar{h}_{k}\left(\vec{x}_{k-1}, x_{k}\right)=\bar{h}_{k}\left(\vec{x}_{k}\right)=h_{k}\left(\vec{z}_{k}\left(\vec{x}_{k}\right)\right)=h_{k}\left(\vec{z}_{k-1}\left(\vec{x}_{k-1}\right), \frac{x_{k}-\mu_{k}\left(\vec{x}_{k-1}\right)}{\sqrt{n} \sigma_{k}\left(\vec{x}_{k-1}\right)}\right)$.

Remind that function $\bar{h}_{k}(\cdot)$ is continuous and bounded, whereas $X_{k}$ and $X_{0}$ are identically distributed. Hence

$$
\overline{\mathbf{E} h} h_{k}\left(\vec{x}_{k-1}, X_{k}\right)=\overline{\mathbf{E}} h_{k}\left(\vec{z}_{k-1}\left(\vec{x}_{k-1}\right), \frac{X_{0}-\mu_{k}\left(\vec{x}_{k-1}\right)}{\sqrt{n} \sigma_{k}\left(\vec{x}_{k-1}\right)}\right)=H_{k}\left(\vec{z}_{k-1}\left(\vec{x}_{k-1}\right)\right) .
$$

In addition we used here definition (84) of $H_{k}(\cdot)$. But $X_{k}$ is independent to $\vec{X}_{k-1}$. Hence using definition (11) of independence with $\psi(\cdot)=\bar{h}_{k}(\cdot)$ we obtain:

$\overline{\mathbf{E}} h_{k}\left(\vec{X}_{k}\right)=\overline{\mathbf{E}}\left[\left.\overline{\mathbf{E}}\left[h_{k}\left(\vec{x}_{k-1}, X_{k}\right)\right]\right|_{\vec{x}_{k-1}=\vec{X}_{k-1}}\right]=\overline{\mathbf{E}} H_{k}\left(\vec{z}_{k-1}\left(\vec{X}_{k-1}\right)\right)=\overline{\mathbf{E}} H_{k}\left(\vec{\zeta}_{k-1}\right)$.

From (92)-94) we have :

$\overline{\mathbf{E}} h_{k}\left(\vec{\zeta}_{k}\right)=\overline{\mathbf{E} h_{k}}\left(\vec{X}_{k}\right)=\overline{\mathbf{E}} H_{k}\left(\vec{\zeta}_{k-1}\right) \leq \overline{\mathbf{E}}\left(h_{k-1}\left(\vec{\zeta}_{k-1}\right)\right)+\tau_{k, n}=\overline{\mathbf{E}} h_{k-1}\left(\vec{\zeta}_{k-1}\right)+\tau_{k, n}$

Here we also used inequality (84) with $\vec{x}_{k-1}=\vec{\zeta}_{k-1}$. So we obtained that $\Delta_{k} \leq$ $\tau_{k, n}$. Substituting the last estimate into (70), we obtain (90) if only we remind that $\Delta_{n+1} \leq 0$ by (73).

Inequality (91) follows immediately from (80) and (85). 
Remark 5. If $X_{1}, X_{2}, \ldots$ are classical random variables then all arguments in the present proof of Theorem 1 remains valid with $\rho=\varkappa=\kappa=0$ for all measurable sets $D$. As a result from (90) with $r=\varepsilon / 2$ we obtain that

$$
\overline{\mathbf{P}}\left(S_{n} \in D\right)-\mathbf{P}\left(B_{n} \in D^{\varepsilon}\right) \leq \frac{16}{\varepsilon^{2}} \mathbf{E} \min \left\{\frac{\left|\xi_{1}\right|^{3}}{\varepsilon \sqrt{n}}, \xi_{1}^{2}\right\}+\frac{16}{\varepsilon^{2}} \mathbf{E} \min \left\{\frac{|Z|^{3}}{\varepsilon \sqrt{n}}, Z^{2}\right\}
$$

where $Z$ has standard normal distribution. This estimate was first obtained in Corollary 3.1 in Sakhanenko [11], using similar, but simplified, arguments.

Estimate (95) may be treated as the first estimate in FCLT with explicit constants. But (95) is not a "real" estimate in FCLT because it is not an estimate for the value:

$$
\Delta_{+}(\varepsilon):=\overline{\mathbf{P}}\left(S_{n} \in D\right)-\mathbf{P}\left(B \in D^{\varepsilon}\right)
$$

which we are going to estimate in the present paper.

4.6. Proof of Theorem [1. We need two auxiliary lemmas.

Lemma 10. If $p \geq 2$ then $\gamma_{p}(C) \geq K_{p}(C):=\min \left\{1,2 C^{p-2} / p\right\}$ for any $C>0$.

Proof. Consider function $f_{0}(x)=\int_{0}^{|x|}(p / 2) \min \left\{y^{p / 2-1}, 1\right\} d y$. It is even and convex because it's derivative $f_{0}^{\prime}(x)$ is non-decreasing for $x \geq 0$. By Jensen inequality for sublinear expectations we have for any $\mu \in[\mu, \bar{\mu}]$ that

$$
\overline{\mathbf{E}} f_{0}\left(\xi_{0}^{2}\right) \geq f_{0}\left(\overline{\mathbf{E}}\left[\xi_{0}^{2}\right]\right)=f_{0}\left(\bar{\sigma}^{2}(\mu) / \underline{\sigma}^{2}(\mu)\right) \geq f_{0}(1)=1,
$$

where $\xi_{0}=\left(X_{0}-\mu\right) / \underline{\sigma}(\mu)$ and $f_{0}(1)=\int_{0}^{1}(p / 2) y^{p / 2-1} d y=1$.

Next consider function $f(x)=\min \left\{|x|^{p / 2}, C^{p-2}|x|\right\}$. It is easy to see that

$$
\forall x \geq 0 \quad f^{\prime}(x)=\left\{\begin{array}{l}
p x^{p / 2-1} / 2, \text { if } x<C^{2} \\
C^{p-2}, \text { if } x>C^{2}
\end{array} \geq K_{p}(C) f_{0}^{\prime}(x) .\right.
$$

Hence, $f(x)=f(|x|) \geq K_{p}(C) f_{0}(|x|)=K_{p}(C) f_{0}(x)$. From this fact, (97), (26) and (27) we obtain that

$$
\gamma_{p}(C) \geq \gamma_{p}(u, C)=\overline{\mathbf{E}} f\left(\xi_{0}^{2}\right) \geq K_{p}(C) \overline{\mathbf{E}} f_{0}\left(\xi_{0}^{2}\right) \geq K_{p}(C) .
$$

Lemma 11. For any $b>0$

$$
\Delta_{n}^{*}:=\mathbf{P}\left(B_{n} \in D^{2 \rho+2 r}\right)-\mathbf{P}\left(B \in D^{2 \rho+2 r+b}\right) \leq \mathbf{P}\left(\left\|B_{n}-B\right\| \geq b\right) \leq \frac{n \mathbf{E}|Z|^{p}}{(\sqrt{n} b)^{p}} .
$$

Proof. It is well known that function $|x|^{p}$ is convex for $p \geq 1$ and $B(t)$ is a martingale. So, by maximal inequality we have

$$
P_{n}^{*}(b):=\mathbf{P}\left(\sup _{t \in[0,1 / n]}|B(t)| \geq b\right) \leq \frac{\mathbf{E}|B(1 / n)|^{p}}{b^{p}}=\frac{\mathbf{E}|B(1)|^{p}}{(\sqrt{n} b)^{p}}=\frac{\mathbf{E}|Z|^{p}}{(\sqrt{n} b)^{p}} .
$$


Note that $B(k / n)=B_{n}(k / n)$ for $k=0,1, \ldots, n$. Hence

$$
\begin{aligned}
\mathbf{P}\left(\left\|B_{n}-B\right\| \geq b\right) & =\mathbf{P}\left(\max _{t \in[0,1]}\left|B_{n}(t)-B(t)\right| \geq b\right) \\
& \leq \sum_{k=1}^{n} \mathbf{P}\left(\sup _{t \in\left[\frac{k-1}{n}, \frac{k}{n}\right]}\left|B_{n}(t)-B\left(\frac{k-1}{n}\right)\right| \geq b\right)=n P_{n}^{*}(b)
\end{aligned}
$$

Since the first inequality in (98) is evident, the result is proved.

Thus, from (90), (98), (96) and (91) with $b=r \leq \varepsilon / 2$ we have:

$$
\Delta_{+}(3 r+2 \rho)=\Delta+\Delta_{n}^{*} \leq \frac{n}{(r \sqrt{n})^{p}}\left[\frac{16 \gamma_{p}(\varepsilon \sqrt{n})}{2^{p}}+\frac{16\left(1+\rho_{0}\right) \mathbf{E}|Z|^{p}}{2^{p}}+\mathbf{E}|Z|^{p}\right] .
$$

Choose $\rho=\rho_{0} \varepsilon / 2$ and $r=\left(\varepsilon-\rho_{0} \varepsilon\right) / 3$ in (99) and assume that $(\varepsilon \sqrt{n})^{p-2} \geq p / 2$. As a result we obtain, by Lemma [10, the desirable inequality (28), but with $C_{5}(p)$ instead of $C_{2}(p)$, where

$$
C_{5}(p):=\frac{3^{p}}{\left(1-\rho_{0}\right)^{p}}\left[\frac{16}{2^{p}}+\frac{16\left(1+\rho_{0}\right) \mathbf{E}|Z|^{p}}{2^{p}}+\mathbf{E}|Z|^{p}\right]
$$

Since $\mathbf{E}|Z|^{p} \leq\left(\mathbf{E}|Z|^{3}\right)^{p / 3}=(4 / \sqrt{2 \pi})^{p / 3}<(1.6)^{p / 3}$, it is not difficult to verify that $C_{5}(p) \leq C_{5}(3)<184$ and that $C_{5}(p) /(4.7)^{p} \leq C_{5}(2) /(4.7)^{2}<4.7$. So, $C_{5}(p) \leq C_{2}(p)$ for all $p \in[2,3]$.

Thus, inequality (28) is proved when $C^{p-2} \geq p / 2$ with $C:=\varepsilon \sqrt{n}$. Consider now the case when $0<C^{p-2}=(\varepsilon \sqrt{n})^{p-2}<p / 2$. Then $C^{2} \leq e p / 2$ and $\gamma_{p}(C) \geq 2 C^{p-2} / p$ by Lemma 10. Hence, in this case the right hand side in (28) has the following property

$$
\frac{C_{2}(p) \gamma_{p}(\varepsilon \sqrt{n})}{n^{(p-2) / 2} \varepsilon^{p}}=\frac{n C_{2}(p) \gamma_{p}(C)}{C^{p}} \geq \frac{2 n C_{2}(p) C^{p-2}}{p C^{p}}=\frac{2 n C_{2}(p)}{p C^{2}} \geq \frac{C_{2}(2)}{e}>1
$$

But the left hand side in (28) does not exceed 1 in view of (22).

So, Theorem 1 is proved in all cases.

4.7. Proofs of Corollaries 1 and 2, We are going to use the assertion of Theorem 1 with $\varepsilon=\min \left\{\tilde{\Pi}_{n, p}, 1\right\}$. In this case $\gamma_{p}(\varepsilon \sqrt{n}) \leq \gamma_{p}(\sqrt{n})$. Hence, for the chosen $\varepsilon$ we have from (28) that $\tilde{\Pi}_{n}(\varepsilon, p) \leq \varepsilon$. Thus, by definition (29), the chosen $\varepsilon$ is an estimate for $\bar{\Pi}\left(S_{n}, B\right)$, and (30) follows.

Now we are going to apply Theorem 1 with the same $\varepsilon$ to set $D=D_{a}=$ $\{x: f(x) \leq a\}$. We obtain

$$
\overline{\mathbf{P}}\left(f\left(S_{n}\right) \leq a\right)=\overline{\mathbf{P}}\left(S_{n} \in D_{a}\right) \leq \mathbf{P}\left(B \in D_{a}^{\varepsilon}\right)+\tilde{\Pi}_{n}(\varepsilon, p) \leq \mathbf{P}\left(B \in D_{a}^{\varepsilon}\right)+\varepsilon .
$$

Since $f(\cdot)$ satisfies condition ([6), we have that $D_{a}^{\varepsilon} \subset D_{a+L \varepsilon}$. Thus, by condition (7) we have

$$
\mathbf{P}\left(B \in D_{a}^{\varepsilon}\right) \leq \mathbf{P}\left(B \in D_{a+L \varepsilon}\right)=\mathbf{P}(f(B) \leq a+L \varepsilon) \leq \mathbf{P}(f(B) \leq a)+K L \varepsilon .
$$

Now (31) follows from (100) and (101). 


\section{Proof of Theorem 2}

5.1. Preliminary considerations. We fix an integer $n \geq 1$, a real number $\varepsilon>0$ and a set $A \subset \mathbb{R}$. Introduce into consideration classical random variable $\nu$ with density $g_{1}(\cdot)$ from (54), which we suppose to be independent from the Wiener process $B$. Underline that $|\nu|<1$ a.s. and define

$$
\tilde{h}_{k}(x):=\mathbf{P}\left(x+\varepsilon \nu / 2+B(1)-B(k / n) \in A^{\varepsilon / 2}\right), \quad k=0,1, \ldots, n .
$$

For any $x, y \in \mathbb{R}$ introduce an error term of the Taylor expansion of $\tilde{h}_{k}(x+y)$ :

$$
\tilde{\delta}_{k}(x, y):=\tilde{h}_{k}(x+y)-\tilde{h}_{k}(x)-y \tilde{h}_{k}^{\prime}(x)-y^{2} \tilde{h}_{k}^{\prime \prime}(x) / 2 .
$$

Lemma 12. If $1 \leq k<n$ then for all $y \in \mathbb{R}$

$$
\tilde{\delta}_{k}(y):=\sup _{x}\left|\tilde{\delta}_{k}(x, y)\right| \leq 0.4|y|^{3}(1-k / n)^{-3 / 2} .
$$

Proof. Note that random variable $Z=(B(1)-B(k / n)) / b$ is independent of $\nu$ and has standard normal distribution when $b=\sqrt{1-k / n}$. Hence, from definition (102) we have that

$$
\tilde{h}_{k}(x)=\mathbf{P}(x+b Z \in A(\varepsilon \nu / 2)) \quad \text { with } \quad A(y)=A^{\varepsilon / 2}-y .
$$

Thus, inequality (104) follows from the assertion (60) of Lemma 4.

5.2. Constructions of $\mu_{k}$ and $\sigma_{k}$. Choose numbers $\varkappa>0$ and $\kappa>0$ so that condition (81) be fulfilled with $\tilde{\delta}_{k}(\cdot)$ instead of $\delta_{k}(\cdot)$. After that define functions

$$
\mu_{k}(x):=\alpha_{\varkappa}(x \mid h) \quad \text { for } \quad h(\cdot)=\tilde{h}_{k}(\cdot), \quad k=2, \ldots, n,
$$

where function $\alpha_{\varkappa}(x \mid h)$ was defined in (36) and (37); and we put

$$
\sigma_{k}(x)=\beta_{\varkappa, \kappa}(x \mid h) \quad \text { for } \quad h(\cdot)=\tilde{h}_{k}(\cdot), \quad k=2, \ldots, n
$$

with function $\beta_{\varkappa, \kappa}(x \mid h)$ introduced in (38) and (39). And for $k=1$ we put

$$
\mu_{1}=\mu_{1}(x)=\underline{\mu} \text { and } \sigma_{1}=\sigma_{1}(x)=\underline{\sigma}(\underline{\mu})>0 .
$$

So, later on in our proof we use only $\zeta_{k}=\left(X_{k}-\mu_{k}\right) / \sigma_{k}$ with $\mu_{k}=\mu_{k}\left(\zeta_{k-1}\right)$ and $\sigma_{k}=\sigma_{k}\left(\zeta_{k-1}\right)$ defined in (105) - (107).

Lemma 13. If all values $\mu_{k}$ and $\sigma_{k}$ are defined in (105) - (107), then

$$
\tilde{\Delta}:=\overline{\mathbf{P}}\left(W_{n} \in A\right)-\mathbf{P}\left(Z \in A^{\varepsilon}\right) \leq \sum_{k=1}^{n} \tilde{\tau}_{k, n},
$$

where

$$
\tilde{\tau}_{k, n} \leq \frac{0.4 C_{7} \gamma_{3}}{(n-k)^{3 / 2}} \quad \text { with } \quad C_{7}:=1+\left(1+\rho_{0}\right) \mathbf{E}|Z|^{3}<2.616,
$$

when $1 \leq k<n$. In addition,

$$
\tilde{\tau}_{k, n} \leq \frac{16}{((\varepsilon-2 \rho) \sqrt{n})^{p}}\left\{\gamma_{p}(\varepsilon \sqrt{n})+\left(1+\rho_{0}\right) \mathbf{E}|Z|^{p}\right\} \leq \frac{16 C_{7} \gamma_{p}}{((\varepsilon-2 \rho) \sqrt{n})^{p}},
$$

for all $k=1, \ldots, n, p \in[2,3]$ and all $\rho \in(0, \varepsilon / 2)$. 
Proof. Consider a partial case of Theorem 1 when

$$
D=\{\tilde{x}(\cdot) \in \mathbb{C}[0,1]: \tilde{x}(1) \in A\} \quad \text { and } r+\rho=\varepsilon / 2 .
$$

In this case $D^{r}=\left\{\tilde{x}(\cdot) \in \mathbb{C}[0,1]: \tilde{x}(1) \in A^{r}\right\}$ for all $r>0$ and hence

$$
\overline{\mathbf{P}}\left(S_{n} \in D\right)=\overline{\mathbf{P}}\left(W_{n} \in A\right), \quad \mathbf{P}\left(B_{n} \in D^{\varepsilon}\right)=\mathbf{P}\left(Z \in A^{\varepsilon}\right),
$$

and $\Delta=\tilde{\Delta}$ as a result. Moreover, it is not difficult to see from definitions (71), (72) and (102) that for all $k=1, \ldots, n$

$$
D_{k}=D^{r+\rho}=D^{\varepsilon / 2} \text { and } h_{k}\left(\vec{x}_{k}\right)=\tilde{h}_{k}\left(x_{1}+\cdots+x_{k}\right) .
$$

Using now definition (79) with (103) and (80) with (104) we obtain that

$$
\delta_{k}\left(\vec{x}_{k-1}, y\right)=\tilde{\delta}_{k}\left(x_{1}+\cdots+x_{k-1}, y\right) \text { and } \delta_{k}(y)=\tilde{\delta}_{k}(y) .
$$

Thus, under assumptions (111), from (112) and (113) we have that assertion (90) of Lemma 9 may be written in the form (108) with

$$
\tilde{\tau}_{k, n}:=\sup _{\mu \in[\underline{\mu}, \bar{\mu}]} \overline{\mathbf{E}}\left[\tilde{\delta}_{k}\left(\xi_{0}(\mu) / \sqrt{n}\right)\right]+\left(1+\rho_{0}\right) \mathbf{E}\left[\tilde{\delta}_{k}(Z / \sqrt{n})\right]=\tau_{k, n} .
$$

Substituting (104) into (114) we obtain (109). At last, (110) is true as a corollary of inequality (91) obtained under more general assumptions.

Note that estimates (104) and (109) are the only facts, used in the proof of Theorem 2 which does not hold under more general conditions of Theorem 1

5.3. Proof of Theorem [2, If $(\varepsilon \sqrt{n})^{p-2} \geq p / 2$ then $\gamma_{p}(\varepsilon \sqrt{n}) \geq 1$ by Lemma 10. In this case substituting estimate (110) into (108) we find inequality (32) with $C_{4}=16 C_{7} /(1-2 \rho / \varepsilon)^{3} \leq 42$ for $\rho$ sufficiently small. When $(\varepsilon \sqrt{n})^{p-2}<p / 2$ we may repeat the arguments used at the end of the proof of Theorem 1 and obtain that (32) again holds because its right hand side is greater than 1.

Let $m$ be the minimal integer not smaller than $x_{0}=n \varepsilon^{2} / 16$. From (109) we have:

$$
\sum_{k<n-m} \tilde{\tau}_{k, n} \leq \sum_{l>m} \frac{0.4 C_{7} \gamma_{3}}{l^{3 / 2}}<\int_{x>m} \frac{1.05 \gamma_{3}}{x^{3 / 2}} d x<\frac{2.1 \gamma_{3}}{x_{0}^{1 / 2}}=\frac{8.4 \gamma_{3}}{\varepsilon \sqrt{n}}
$$

On the other hand, from (110) with $p=3$ for sufficiently small $\rho>0$ we obtain:

$$
\sum_{k \geq n-m}^{n} \tilde{\tau}_{k, n} \leq \frac{(m+1) 16 C_{7} \gamma_{3}}{((\varepsilon-2 \rho) \sqrt{n})^{3}} \leq\left(x_{0}+2\right) \frac{16 \cdot 2.7 \gamma_{3}}{(\varepsilon \sqrt{n})^{3}}=\frac{2.7 \gamma_{3}}{\varepsilon \sqrt{n}}+2 \frac{16 \cdot 2.7 \gamma_{3}}{(\varepsilon \sqrt{n})^{3}} .
$$

Substituting estimates (115) and (116) into (108) we obtain (33) in the case when $\varepsilon \sqrt{n} \geq 12$. But if $\varepsilon \sqrt{n}<12$, then right hand side in (33) greater than 1 . So, (33) takes place in all cases.

Thus, all assertions of the paper are proved.

Acknowledgments The authors are grateful to the anonymous referee for his/her thorough review and highly appreciate his/her comments, which inspired us to improve the results and their presentation. 


\section{References}

[1] A. A. Borovkov, "On the rate of convergence for the invariance principle", Theory Probab. Appl., 18:2 (1974), 207-225.

[2] M. D. Donsker, "An invariance principle for certain probability limit theo-rems", Memoirs of the American Mathematical Society, 6:6 (1951), 885-900.

[3] Xiao Fang, Shige Peng, Qi-man Shao, Yongsheng Song, Limit theorems with rate of convergence under sublinear expectations (2017), arXive: 1711.10649v1 (accepted to Bernoulli).

[4] Shige Peng, "Nonlinear expectations and nonlinear Markov chains", Chinese Ann. Math. Ser. B, 26:2 (2005), 159-184.

[5] Shige Peng, "G-Expectation, G-Brownian Motion and Related Stochastic Calculus of Ito's type", Stochastic Analysis and Applications, 2:4 (2006), $541-567$.

[6] Shige Peng, "A New Central Limit Theorem under Sublinear Expectations", Math, 53:8 (2008), 1989-1994.

[7] Shige Peng, "Nonlinear Expectations and Stochastic Calculus under Uncertainty" (2010), arXiv:1002.4546v1

[8] Yu. V. Prokhorov, "Convergence of random processes and limit theorems in probability theory", Theory Probab. Appl., 1:2 (1956), 157-214.

[9] A. I. Sakhanenko, "Simple method of obtaining estimates in the invariance principle", Probability Theory and Mathematical Statistics, Springer Berlin Heidelberg (1988), 430-443.

[10] A. I. Sakhanenko, "On the accuracy of normal approximation in the invariance principle", Proceedings of the Institute of Mathematics Novosibirsk, 13 (1989), 40-66. (English translation in: Siberian Advances in Mathematics, 1:4 (1991), 58-91).

[11] A. I. Sakhanenko, "A new way to obtain estimates in the invariance principle", High dimensional probability II. Progr. Probab, 47 (2000), 223-245, Birkhäuser Boston.

[12] A. I. Sakhanenko, "Estimates in the invariance principle in terms of truncated power moments", Siberian Math. J., 47:6 (2006), 1113-1127.

[13] V. V. Yurinskii, "A smoothing inequality for estimations of Levy Prokhorov distance", Theory Probab. Appl., 20:1 (1975), 1-10. 